\title{
Retrokonversion des Alphabetischen Zettelkatalogs erfolgreich abgeschlossen
}

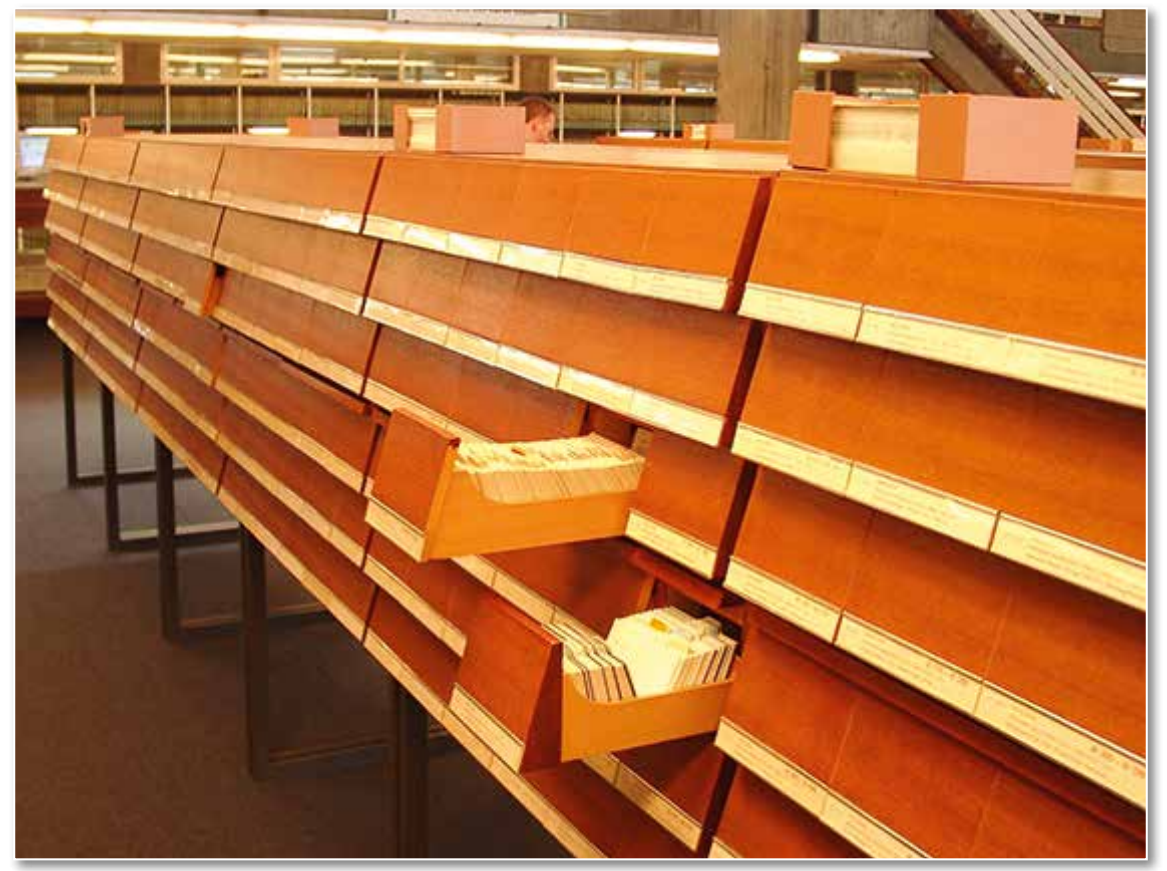

\section{Geschafft!}

Seit Ende 2016 ist der Alphabetische Zettelkatalog der Württembergischen Landesbibliothek gänzlich retrokonvertiert. Nach hausinternen Konversionsprojekten sowie nach einer fast sechsjährigen Konversion mit der Firma BBI liegen die bibliographischen Metadaten vollständig maschinenlesbar vor. Der Alphabetische Zettelkatalog war das einzige umfassende Nachweisinstrument für den nicht nur sehr umfangreichen, sondern auch wertvollen und bedeutenden Bibliotheksbestand mit Erscheinungsjahren von 1501 bis 1988. Der Katalog umfasste 2.700 Katalogkästen mit knapp 2,7 Mio. Katalogzetteln. Seit 2004 wurde der Zettelkatalog für die Benutzung über die Homepage der Bibliothek als digitalisierter Katalog, als "DigiKat", angeboten. Eine Beschreibung der unterschiedlichen WLB-Konversionsprojekte und des Konversionsverfahrens mit einer Firma erfolgte in WLBforum, 14 (2012), Heft 2, S. 9-12 unter dem Titel "Der Alphabetische Zettelkatalog - Retrokonversion durch eine Firma".

Der Alphabetische Zettelkatalog verzeichnete Monographien, mehrbändige Werke, gezählte Serien sowie Zeitschriften, zeitschriftenartige Reihen und Zeitungen. Katalogisiert waren das "Alte und Wertvolle Buch" sowie das "Moderne Buch". Ganz überwiegend waren in dem Katalog Druckwerke nachgewiesen, aber er enthielt auch Nachweise von Nicht-Buch-Materialien wie Schallplatten, Mikrofiches, Mikrofilme, Disketten und CDs.

\section{Vereinfachung und Verbesserung der Recherche für die Benutzer}

All diese Werke wurden bis Ende des Jahres 2016 im SWB oder in der ZDB retrospektiv erfasst und sind nunmehr einheitlich im OPAC recherchierbar. Die Benutzer können jetzt, egal für welchen Bestandteil sie sich interessieren, komfortabel mit einer Suchmaske in einer Datenbank recherchieren bzw. suchen und finden. Für die Benutzung ist es mit der Realisierung eines einzigen Datenpools irrelevant geworden, welche Materialart welcher Erscheinungsjahre nach welchen Katalogregeln erfasst wurde. Das Rechercheinstrument ist der Online-Katalog (OPAC) der WLB und nicht mehr unterschiedliche digitalisierte Zettelkataloge. 


\section{Zugewinn für die bibliothekarische Arbeit}

Der einheitliche Datenpool bringt darüber hinaus Vorteile für künftige bibliothekarische Projekte. Die hauseigenen bibliographischen Metadaten können nun sowohl vollständig als auch gezielt ausgewertet und separiert bearbeitet werden. Beispielsweise Änderungen von Benutzungsmerkmalen, ausgewählte Restaurierungsmaßnahmen oder spezielle Abfragen zum Pflichtbestand könnten durch definierte Suchanfragen über den Datengesamtbestand vorbereitet werden.

Ebenso können erst seit Abschluss der Retrokonversion technische Vorteile eines integrierten Bibliothekssystems - nämlich alle relevanten Daten aus Erwerbung, Katalogisierung und Benutzung sind Teil eines einzigen Lokalsystems - wirksam werden.

\section{Umfang der WLB-Metadaten in SWB und ZDB}

In der Statistik des Südwestdeutschen Bibliotheksverbundes sind für die WLB mit Stand 31.12.2016 insgesamt 3.280.223 Lokalsätze nachgewiesen. Die Retrokonversion mit der Firma BBI lief über fast sechs Jahre von 2011 bis 2016. In diesem Konversionsprojekt wurden im SWB ca. 951.000 Titelaufnahmen angelegt bzw. Ansigelungen vorgenommen, d. h. von den insgesamt 3.280.223 WLB-Lokalsätzen im SWB stammen mit Stand Ende 2016 etwa 29\% aus der Retrokonversion mit der Firma.

Die SWB-Titelaufnahmen und Ansigelungen aus den hauseigenen Konversionsprojekten ergeben eine Zahl von ca. 686.000. Addiert man diese und die aus dem Firmenkonversionsprojekt, dann kann man errechnen, dass von den insgesamt 3.280.223 WLB-Lokalsätzen im SWB mit Stand Ende 2016 fast $50 \%$ aus diversen Konversionsprojekten stammen.

Die Zahl der Titelnachweise in der ZDB mit Stand 31.12.2016 umfasst 174.727. Im Zusammenhang mit der Retrokonversion durch die Firma wurden von bibliothekarischen Stammkräften in der ZDB anhand der gemeldeten Titelkarten in 447 Fällen Zeitschriften-Titelaufnahmen neu angelegt sowie in 4.614 Fällen Lokalsätze angehängt und Bestandseinträge neu aufgenommen.

\section{Konversion mit einer Firma, aber nicht ohne Bibliothekare}

Die Konversionsfirma hat Abertausende von Scans ohne und mit Recherche im SWB überprüft, sie hat an vorhandene Titelsätze Abertausende von Lokalsätzen angehängt, sie hat viele Tausende Titelsätze aus Fremddaten übernommen, und sie hat viele Tausende neue Titelsätze im SWB angelegt. Und doch wäre eine Retrokonversion nur durch eine Firma und gänzlich ohne Bibliothekarinnen und Bibliothekare nicht durchführbar gewesen.

Die Konversion durch eine Firma musste mit bibliothekarischer Sachkenntnis vorbereitet werden. Die Anlage des Katalogs und seiner Regeln waren zu ermitteln und für die Firma in Erfassungsanleitungen umzusetzen. Ausschreibungen und Auftragsvergaben mussten durchgeführt werden. Und während der gesamten Laufzeit mussten Datenlieferungen und Rechnungen geprüft werden. Nachdem die Erfassung durch die Firma begonnen hatte, wurden der Bibliothek während der gesamten Projektlaufzeit die Scans gemeldet, die schlichtweg nicht lesbar, die ohne Kenntnis der hauseigenen Katalogregeln nicht zu bearbeiten waren oder deren Katalogisierung ohne Autopsie nicht realisiert werden konnte. Diese Rückmeldungen hatten einen Umfang von mehreren Zehntausend und mussten während des gesamten Projekts zeitnah von mehreren Bibliothekarinnen und Bibliothekaren der WLB abgearbeitet werden.

\section{Weitere Retrokonversionsvorhaben}

Unmittelbar im Anschluss begann im Januar 2017 die Retrokonversion des Musik-AK, aus dem gedruckte Noten nach RDA im SWB erfasst werden sollen. Der Musik-AK umfasst lediglich ca. 155.000 Scans, und noch zu erfassen sind ausschließlich Notendrucke, so dass im Jahr 2017 mit einem Abschluss des Projekts zu rechnen ist. Die beauftragte Firma ist wiederum BBI.

Vorbereitet wird zudem seit Anfang 2017 die Retrokonversion des Alphabetischen Zettelkatalogs der Bibliothek für Zeitgeschichte, die seit dem Jahr 2000 integraler Bestandteil der Württembergischen Landesbibliothek ist. Der Alphabetische Zettelkatalog der Bibliothek für Zeitgeschichte enthält ca. 338.000 Scans und hat seine ganz eigenen Katalogisierungs- und Erfassungsmodalitäten. Die Erfassung durch eine Firma kann voraussichtlich Mitte 2018 beginnen.

Angelika Schütt-Hohenstein 